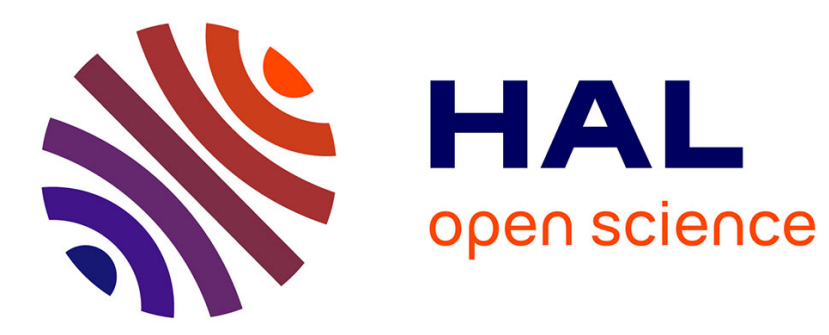

\title{
Model Reduction Based Approximation of the Output Controllability Gramian in Large-Scale Networks
}

Giacomo Casadei, Carlos Canudas de Wit, Sandro Zampieri

\section{To cite this version:}

Giacomo Casadei, Carlos Canudas de Wit, Sandro Zampieri. Model Reduction Based Approximation of the Output Controllability Gramian in Large-Scale Networks. IEEE Transactions on Control of Network Systems, In press, 10.1109/TCNS.2020.3000694 . hal-02883901

\section{HAL Id: hal-02883901 https://hal.science/hal-02883901}

Submitted on 29 Jun 2020

HAL is a multi-disciplinary open access archive for the deposit and dissemination of scientific research documents, whether they are published or not. The documents may come from teaching and research institutions in France or abroad, or from public or private research centers.
L'archive ouverte pluridisciplinaire $\mathbf{H A L}$, est destinée au dépôt et à la diffusion de documents scientifiques de niveau recherche, publiés ou non, émanant des établissements d'enseignement et de recherche français ou étrangers, des laboratoires publics ou privés. 


\title{
Model Reduction Based Approximation of the Output Controllability Gramian in Large-Scale Networks
}

\author{
Giacomo Casadei, Carlos Canudas-de-Wit and Sandro Zampieri
}

\begin{abstract}
In this paper we consider the problem of determining the control energy for large-scale networks. Instead of controlling all the nodes of the network, we are interested in driving the value of some outputs to the desired value, by controlling directly some of the nodes. For doing this, we exploit the concept of output controllability and of output controllability Gramian that permits to analyze the properties of the system, both in single-output and multi-output case. Based on a projection method, we show that it is possible to obtain an approximated model which makes the computation of the Gramian and its interpretation much easier. Simulations show that the reduced model is consistent with the original one and provides a reliable approximation of the control energy necessary to control the network.
\end{abstract}

\section{INTRODUCTION}

The interest in the control of networks is motivated by the vast number of applications which can be interpreted as networks of different natures. Robot swarms [2], power networks [3] and social networks [4], are few examples which motivated researchers to study control problems for networks. Many results about consensus and synchronization can be found both in the context of linear [5], [6] and nonlinear systems [7], [8]. Due to the increasing size and complexity of network applications, many researchers started to focus on large-scale systems. The analysis of large-scale networks has been widely covered in the literature and notable contributions can be found in [9], [10], [11].

The problem of controlling large-scale networks is to a great extent still open. Controllability of a network [12] relates to the possibility of driving the network state to a desired value by acting on the driver nodes that are called inputs. Instead, structural controllability [13] concerns this property when only the nonzero pattern of the network adjacency matrix is given. Controllability and structural controllability for large-scale networks, and in particular the minimum number of control inputs that makes the network controllable,

G. Casadei is with Laboratoire Ampere Dpt. EEA of the École Centrale de Lyon, Université de Lyon, 69134 Ecully, France, C. Canudas-de-Wit are with Univ. Grenoble Alpes, CNRS, Inria, GIPSA-lab, F-38000 Grenoble, France. S. Zampieri is with Università di Padova. The research leading to these results has received funding from the European Research Council (ERC) under the European Union's Horizon 2020 research and innovation program (grant agreement $\mathrm{N}^{\circ}$ 694209). Part of this work was supported by MIUR (Italian Minister for Education) under the initiative "Departments of Excellence" (Law 232/2016). Preliminary results have been presented in the conference paper [1]. has been treated by several authors in recent years (see for instance [14], [15]).

A key point that is particularly important in this context concerns the energy needed for the control. In fact, only when the energy needed to control is limited, we can say that control is an operation that is possible not only in principle, but also in practice. Hence, in this case we can say that we have practical controllability. It has been shown that for large-scale networks there might be cases in which we have exact controllability, but not practical controllability, because the energy needed to control is not realistic [16]. It has been shown that several aspects, such as the number of control nodes [17], the real part of the eigenvalues of the network matrix [18], its centrality measures [19], [20], or its distance to normality [20] play a fundamental role in assessing practical controllability. These results are obtained with the analysis of the controllability Gramian matrix [21]. The latter can be obtained by solving a Lyapunov equation which is a linear equation in $n^{2}$ variables, where $n$ is the network dimension. Even if there are numerically efficient methods for solving this equation exploiting the sparsity of the network [22], this computation remains challenging for large dimensional networks.

One way to simplify the problem consists in replacing the conventional controllability with the concept of output controllability. Precisely, controllability may be not a realistic requirement, since driving the entire state of a large-scale network to an arbitrary final value may be a too stringent requirement. It appears more realistic to drive only a function of the state to a desired value. This function is called the output of the system. The possibility to drive this output arbitrarily is called output controllability, while the energy required to perform this operation is determined by the output controllability Gramian. By its definition, however, the output controllability Gramian still requires the controllability Gramian and thus presents the computational problems mentioned previously. To overcome this issue, in this paper we propose a method for computing an approximation of the output controllability Gramian, relying on an output based model reduction. In particular, we define a reduced model which aggregates the dynamics which contributes to the outputs. Other authors considered the possibility to exploit modelreduction techniques to simplify the problem of controlling a large-scale network: between the others, in [23] the authors introduced different model reduction techniques (based on 

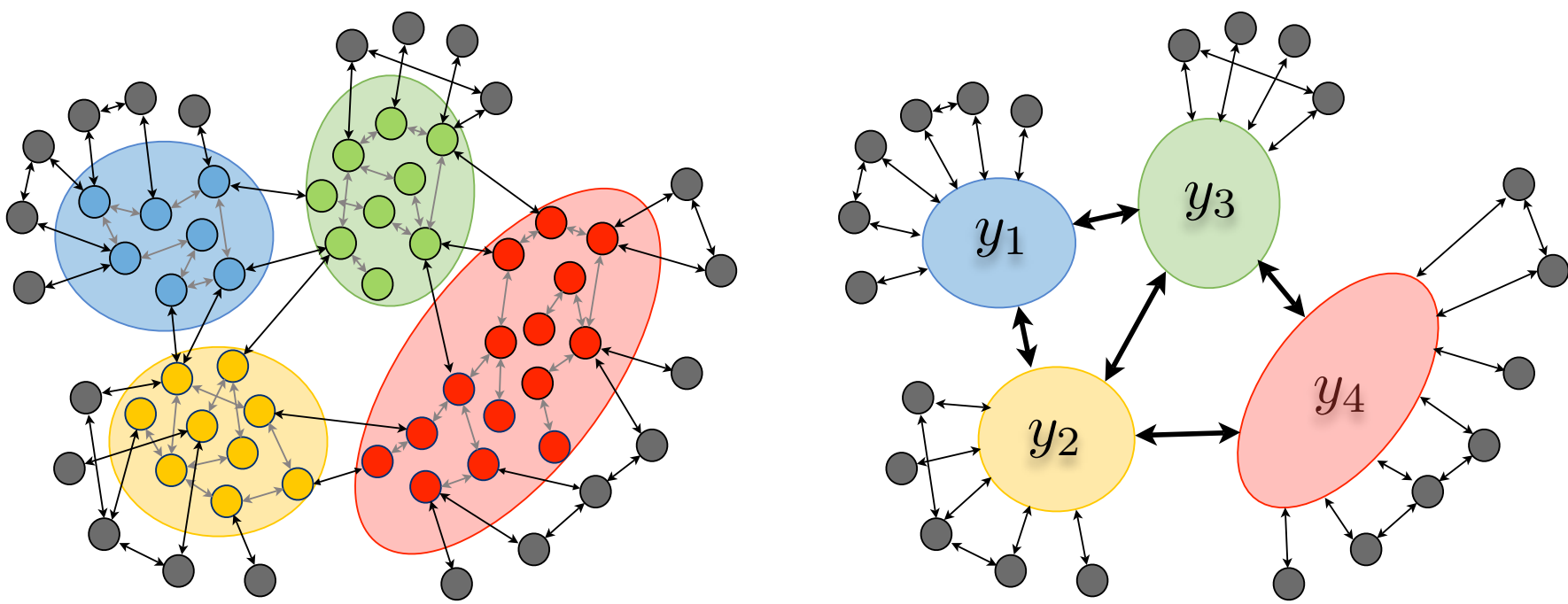

Fig. 1: Schematic of the framework: each partition is composed by nodes which contributes to a certain measure. Partitions are controlled by boundary nodes and interact with each other.

clusters of the networks) to obtain a simplified representation of the original dynamics. Another interesting approach has been presented in [24], where the authors considers PetrovGalerkin projection techniques to achieve a partition-based complexity reduction.

The problem we consider is inspired by two consideration. On one hand, in many practical cases networks are partitioned by the physics of the systems (weak couplings between areas of a network) and the measurements are available for these partitions (for instance, average quantity of a certain zone). On the other hand, often it is not possible to select arbitrarily the controlled nodes: this is case in some notable applications (e.g. traffic network, deep brain stimulation, heating networks) where controlled nodes seat on the boundary of the network. In this context schematically represented in Figure 1, partitions are defined by the output measures (aggregate measures of zones) which are controlled by a set of boundary nodes (black nodes in the figure).

In this paper we consider the case of a network of first order systems and we propose a projection-based model reduction based on the outputs. We show that, from an energy perspective, the approximated model is still consistent with the original one. In fact, from this reduced model it is possible to compute an approximated output controllability Gramian of the original system with a drastic reduction of the computational complexity and with an improved numerical stability. Furthermore, we will also be able to draw some conclusions on the impact of the network structure and on the controllability. Preliminary results were presented in [1], where we focused on the MISO case (multi-input singleoutput).

The paper is structured as follows. In Section II, we present fundamental facts about controllability and output controllability of linear systems. In Sections III we tackle the problem for MISO systems. In Section IV we consider the more challenging case of MIMO (multi-input multi-output) systems where we extend our approach to a more general scenario in which a dynamic controller is required in order to achieve a decoupling between the partitions.

\section{PROBlem Formulation}

Consider a network represented by a directed graph, that is a triplet $\mathcal{G}=(\mathcal{V}, \mathcal{E}, A)$ in which:

- $\mathcal{V}$ is the set of $n$ nodes, $\mathcal{V}=\{1,2, \ldots, n\}$, one for each of the $n$ agents.

- $\mathcal{E} \subset \mathcal{V} \times \mathcal{V}$ is the set of edges that models the interconnection between the agents.

- $A \in \mathbb{R}^{n \times n}$ is the weighted adjacency matrix such that $A_{i j}=0$ whenever $(i, j) \notin \mathcal{E}$. This matrix describes the strength of the interaction between agents.

For each time $t \in \mathbb{R}^{+}$and with each node $i \in \mathcal{V}$ we associate a real value $x_{i}(t)$ representing its state at time $t$. We collect the agents states into a vector $x(t) \in \mathbb{R}^{n}$. The dynamics of the network with inputs is described by the linear and time-invariant differential equation

$$
\dot{x}(t)=A x(t)+B u(t),
$$

where $u(t) \in \mathbb{R}^{m}$ is the input which is the way in which we can influence the state evolution and $B \in \mathbb{R}^{n \times m}$. We assume in the sequel that $A \in \mathbb{R}^{n \times n}$ is Hurwitz stable.

Remark 1 Compared to the models proposed in [25], in this paper we consider a simplified model of dynamic network. Indeed, we assume that each agent dynamics is a first order differential equation. Although less general than the models proposed in [26], the model we propose is general enough to cover many important applications such as diffusive systems with some dissipation term that makes them Hurwitz For instance, transportation networks where the dynamics of the nodes describes a conservation law (reservoir of the network) with some additional dissipation terms (sinks of the network) 
[27]. Less restrictive assumptions on the network dynamics and on the nodes dynamics are left for future work.

The dynamic network (1) is said to be controllable if it is possible to drive its state from any initial value $x(0)$ to any final value $x_{f}$ at time $t_{f}>0$ by a suitable choice of the input $u(\tau), \tau \in\left[0, t_{f}\right]$. It is well-known that (1) is controllable if and only if the $n \times n m$ matrix $\mathcal{C}=$ $\left[\begin{array}{lllll}B & A B & A^{2} B & \ldots & A^{n-1} B\end{array}\right]$ is full row rank.

Another condition ensuring controllability is related to the controllability Gramian which is defined as

$$
\mathcal{W}=\int_{0}^{t_{f}} e^{A \tau} B B^{T} e^{A^{T} \tau} \mathrm{d} \tau .
$$

It is well-known that (1) is controllable if and only if the controllability Gramian is an invertible matrix. The controllability Gramian allows also to compute the cost of the control in terms of the energy needed. Indeed, the energy necessary to steer the system from $x(0)=0$ to a desired final value $x(t)=x_{f}$ is given by

$$
E\left(x_{f}, t_{f}\right)=\left\|u_{\text {opt }}(\cdot)\right\|_{\mathcal{L}_{2}}^{2}=x_{f}^{T} \mathcal{W}^{-1} x_{f},
$$

where $u_{\text {opt }}(\cdot)$ is the optimal input achieving the control objective while minimizing the control energy and where $\left\|u_{\text {opt }}(\cdot)\right\|_{\mathcal{L}_{2}}$ is the $\mathcal{L}_{2}$-norm, namely

$$
\left\|u_{\text {opt }}(\cdot)\right\|_{\mathcal{L}_{2}}=\sqrt{\int_{0}^{t_{f}}\left\|u_{\text {opt }}(\tau)\right\|^{2} \mathrm{~d} \tau} .
$$

Different controllability metrics can be derived from the controllability Gramian:

- Worst case energy: It can be shown that

$$
\max _{\left\|x_{f}\right\|=1} E\left(x_{f}, t_{f}\right)=\frac{1}{\lambda_{\min }(\mathcal{W})},
$$

where $\lambda_{\min }(\mathcal{W})$ is the minimum eigenvalue of $\mathcal{W}$. This implies that, the larger $\lambda_{\min }(\mathcal{W})$, the more controllable the system is.

- Average control energy: If we assume that $x_{f}$ is uniformly distributed in the unit sphere, then it can be shown that $\mathbb{E}\left[E\left(x_{f}, t_{f}\right)\right]=\operatorname{Tr}\left(\mathcal{W}^{-1}\right)$ where $\mathbb{E}[\cdot]$ means the expected value. This implies that, the smaller $\operatorname{Tr}\left(\mathcal{W}^{-1}\right)$, the more controllable the system is.

- Size of the controllable set: The determinant of $\mathcal{W}$ gives the volume of the hyperellipsoid of final states $x_{f}$ that are reachable from the initial state $x(0)=0$ and with control input energy $E\left(x_{f}, t_{f}\right) \leq 1$.

Given a certain network matrix $A$, and an input matrix $B$, we often have that the associated dynamic network (1) is controllable. However it could be that the network is practically not controllable because the energy needed to perform the control task might be unrealistic. The analysis of the controllability Gramian plays a fundamental role in assessing if a system is practically controllable or not. However, for large-scale networks, the calculation of the Gramian (2) is computationally demanding and typically suffers of numerical errors and ill-conditionement [28], [29]. The computation of the metrics introduced before is thus a delicate task subject to computational inaccuracies. With respect to this problem, it is worth pointing out that model reduction techniques may help in addressing the computational issues mentioned above: by reducing the size of the system, the computation of the Gramian and its metrics become less critical at the price of a reduced accuracy.

\section{A. Output controllability and the $\mathcal{H}_{2}$-norm}

In many applications involving large-scale networks, controlling the entire state is an unrealistic task. For this reason, in this paper we are interested in controlling an output of the system rather than its full state. A dynamic network with input and output is given by

$$
\Sigma:\left\{\begin{array}{l}
\dot{x}(t)=A x(t)+B u(t) \\
y(t)=C x(t)
\end{array},\right.
$$

where $y(t) \in \mathbb{R}^{r}$ and $C \in \mathbb{R}^{r \times n}$. This is said to be output controllable if for any $y_{f} \in \mathbb{R}^{r}$ at time $t_{f}>0$ there exists an input function $u(\tau), \tau \in\left[0, t_{f}\right]$, such that the output is driven to $y\left(t_{f}\right)=y_{f}$ assuming that $x(0)=0$. This property can be checked through the rank of the output controllability matrix (see [30]).

Lemma 1 System (4) is output controllable if and only if the $r \times n m$ matrix

$$
\mathcal{C}_{\mathcal{O}}=\left[\begin{array}{lllll}
C B & C A B & C A^{2} B & \ldots & C A^{n-1} B
\end{array}\right]
$$

has full row rank.

The energy necessary to steer the output to a desired final value $y\left(t_{f}\right)=y_{f}$, assuming that $x(0)=0$, can be expressed as a function of the output controllability Gramian

$$
\mathcal{W}_{o}=C \mathcal{W} C^{T}
$$

Lemma 2 Given the dynamic network (4), the minimal energy necessary to steer the output to a desired final value $y(t)=y_{f}$, assuming that $x(0)=0$, is given by

$$
E\left(y_{f}, t_{f}\right)=y_{f}^{T} \mathcal{W}_{o}^{-1} y_{f}
$$

where $\mathcal{W}_{o}$ is given in (5). The optimal input is

$$
u_{o p t}(\tau)=B^{T} e^{A^{T}\left(t_{f}-\tau\right)} C^{T} \mathcal{W}_{o}^{-1} y_{f}
$$

Proof: The proof is a straightforward extension of [31, pag. 160].

The analysis of the output controllability Gramian defined in (5) allows to understand the input-output energy relationship for system (4). However at this stage, the output controllability Gramian $\mathcal{W}_{o}$ still requires the computation of the controllability Gramian $\mathcal{W}$. In order to overcome the issues concerning the computation of the Gramian for large-scale systems, in Section III we introduce a reduced model which allows to simplify the calculation of the output controllability Gramian. As shown in [32, Cap. 5], the output controllability Gramian can be used to compute the 
performance of a system. In fact, it is possible to express the $\mathcal{H}_{2}$-norm of the linear stable system (4) as follows

$$
\|\Sigma\|_{\mathcal{H}_{2}}=\sqrt{\operatorname{trace}\left[\mathcal{W}_{o}\right]} .
$$

We recall that the $\mathcal{H}_{2}$-norm of a system gives both the RMS (root mean square) response to white noise and the $\mathcal{L}_{2}$ norm of the impulse response matrix of the system, namely

$$
\|\Sigma\|_{\mathcal{H}_{2}}=\sqrt{\int_{0}^{\infty} \operatorname{trace}\left[g(t) g(t)^{T}\right] \mathrm{d} t},
$$

where $g(t)=C e^{A t} B$.

\section{A REDUCED MODEL NETWORK: THE CASE OF SINGLE} OUTPUT

In this section, we are interested in studying a particular class of systems in the form

$$
\begin{aligned}
\dot{x} & =A x+B u \\
y & =C x
\end{aligned}
$$

in which the output is scalar and so $C \in \mathbb{R}^{1 \times n}$. In the applications we have in mind, $A \in \mathbb{R}^{n \times n}$ is a Hurwitz Meltzer matrix, $B \in \mathbb{R}^{n \times m}$ is the input matrix which defines which nodes are controlled and it is defined as a $\{1,0\}$ 's entries matrix. We assume moreover that the scalar output depends only on the uncontrolled nodes.

By reordering the nodes in a suitable way, (9) can be rewritten as

$$
\begin{aligned}
{\left[\begin{array}{c}
\dot{z} \\
\dot{\delta}
\end{array}\right] } & =\left[\begin{array}{ll}
A_{11} & A_{12} \\
A_{21} & A_{22}
\end{array}\right]\left[\begin{array}{l}
z \\
\delta
\end{array}\right]+\left[\begin{array}{c}
I_{m} \\
0
\end{array}\right] u, \\
y & =\left[\begin{array}{ll}
0_{1 \times m} & H^{T}
\end{array}\right]\left[\begin{array}{l}
z \\
\delta
\end{array}\right]
\end{aligned}
$$

in which $z=\left[x_{1}, x_{2}, \ldots, x_{m}\right]^{T}$ refers to the nodes which are controlled directly, $\delta=\left[x_{m+1}, x_{m+2}, \ldots, x_{n}\right]^{T}$ refers to the nodes which contributes to the output through $H \in \mathbb{R}^{n-m}$. Conversely we can say that the output is some aggregate measure of the uncontrolled nodes. The sum or the average of their values are two important instances of the outputs that are relevant in the applications that the paper aims to address [34].

In order to simplify the computation of the output controllability Gramian and of the minimal energy control input, we define a reduced model for (10) by expressing the dynamics of the uncontrolled nodes as a scalar dynamics related to the evolution of the output. To this end, we define a matrix $P \in \mathbb{R}^{(m+1) \times n}$ and a new state $\hat{x} \in \mathbb{R}^{m+1}$

$$
P=\left[\begin{array}{cc}
I_{m} & 0 \\
0 & H^{T}
\end{array}\right], \quad \hat{x}=P x .
$$

From (10), we can write

$$
\begin{aligned}
\dot{\hat{x}} & =\left[\begin{array}{cc}
A_{11} & A_{12} \\
H^{T} A_{21} & H^{T} A_{22}
\end{array}\right] x+\left[\begin{array}{c}
I_{m} \\
0
\end{array}\right] u \\
& =\left[\begin{array}{c}
A_{11} \\
H^{T} A_{21}
\end{array}\right] z+\left[\begin{array}{c}
A_{12} \\
H^{T} A_{22}
\end{array}\right] \delta+\left[\begin{array}{c}
I_{m} \\
0
\end{array}\right] u
\end{aligned} .
$$

Furthermore, if we define

$$
P^{+}=\left[\begin{array}{cc}
I_{m} & 0 \\
0 & \frac{1}{\sigma} H
\end{array}\right] \in \mathbb{R}^{n \times(m+1)},
$$

where $\sigma=H^{T} H \in \mathbb{R}$, then we have

$$
P P^{+}=\left[\begin{array}{cc}
I_{m} & 0 \\
0 & 1
\end{array}\right] \quad P^{+} P=\left[\begin{array}{cc}
I_{m} & 0 \\
0 & \frac{1}{\sigma} H H^{T}
\end{array}\right] .
$$

From this we can argue that

$$
\begin{aligned}
x & =P^{+} \hat{x}+\left(I_{n}-P^{+} P\right) x \\
& =\left[\begin{array}{cc}
I_{m} & 0 \\
0 & \frac{1}{\sigma} H
\end{array}\right] \hat{x}+\left[\begin{array}{cc}
0 & 0 \\
0 & I_{\eta}-\frac{1}{\sigma} H H^{T}
\end{array}\right]\left[\begin{array}{l}
z \\
\delta
\end{array}\right],
\end{aligned}
$$

where we set $\eta=n-m$ and we obtain

$$
\delta=\left[\begin{array}{ll}
0 & \frac{1}{\sigma} H
\end{array}\right] \hat{x}+\left(I_{\eta}-\frac{1}{\sigma} H H^{T}\right) \delta .
$$

Thus (12) can be rewritten as

$$
\begin{aligned}
\dot{\hat{x}}=\left[\begin{array}{cc}
A_{11} & \frac{1}{\sigma} A_{12} H \\
H^{T} A_{21} & \frac{1}{\sigma} H^{T} A_{22} H
\end{array}\right] \hat{x}+\left[\begin{array}{c}
I_{m} \\
0
\end{array}\right] u \\
+\left[\begin{array}{c}
A_{12}\left(I_{\eta}-\frac{1}{\sigma} H H^{T}\right) \\
H^{T} A_{22}\left(I_{\eta}-\frac{1}{\sigma} H H^{T}\right)
\end{array}\right] \delta
\end{aligned}
$$

System (13) is a reduced model for (10) and in compact form can be written as

$$
\begin{aligned}
\dot{\hat{x}} & =\hat{A} \hat{x}+\hat{B} u+\hat{D} \delta \\
y & =\hat{C} \hat{x}
\end{aligned}
$$

Note that, if $\hat{D}=\mathbf{0}_{(m+1) \times \eta}$, then the output controllability Gramian $\mathcal{W}_{o}$ of the original system coincides with the output controllability Gramian $\hat{\mathcal{W}}_{o}$ of the reduced system (14). However, the case of $\hat{D}=\mathbf{0}_{(m+1) \times \eta}$, commonly referred to as exact projection, holds only in particular cases (see [33] for more details on the subject). In [34], the authors provide an extensive study of the conditions under which applying (11) to a network leads to $\hat{D}=\mathbf{0}_{(m+1) \times \eta}$.

If we neglect the effect of $\delta$ on $\hat{x}$, the new system

$$
\dot{\hat{x}}=\left[\begin{array}{cc}
A_{11} & \frac{1}{\sigma} A_{12} H \\
H^{T} A_{21} & \frac{1}{\sigma} H^{T} A_{22} H
\end{array}\right] \hat{x}+\left[\begin{array}{c}
I_{m} \\
0
\end{array}\right] u
$$

is only an approximation of the original system and hence $\mathcal{W}_{o}$ and $\hat{\mathcal{W}}_{o}$ will be different. We are interested in comparing these two output controllability Gramians.

Before doing so and to simplify the computations of the output controllability Gramian of the reduced system, we can do other manipulations. In general the top-right term $\frac{1}{\sigma} A_{12} H$ in $\hat{A}$ may be nonzero, namely the controlled nodes can be influenced by the aggregated uncontrolled nodes. If this is the case, we can define

$$
u=\left[\begin{array}{ll}
0 & -\frac{1}{\sigma} A_{12} H
\end{array}\right] \hat{x}+v=-\frac{1}{\sigma} A_{12} H y+v
$$

in such a way that (15) becomes

$$
\dot{\hat{x}}=\left[\begin{array}{cc}
A_{11} & 0 \\
H^{T} A_{21} & \frac{1}{\sigma} H^{T} A_{22} H
\end{array}\right] \hat{x}+\left[\begin{array}{c}
I_{m} \\
0
\end{array}\right] v .
$$


Notice that, if we apply an input $v$ to the system (17), this will generate a state evolution $\hat{x}$ and a corresponding output evolution $y$. If we apply the input $u$ obtained from $\hat{x}$ and $v$ by (16) to the system (15), this will generate the same state evolution $\hat{x}$ and output evolution $y$. However, the energy of the inputs $u$ and $v$ will be different. The following lemma provides an estimation of this difference and an estimation of the difference between the two output controllability Gramians.

Lemma 3 Let $v$ be an input generating a state evolution $\hat{x}$ of the system (17) and let $u$ in (16) be generated from these $\hat{x}$ and

upsilon. Then

$$
\|u(\cdot)\|_{\mathcal{L}_{2}} \leq(1+\gamma)\|v(\cdot)\|_{\mathcal{L}_{2}},
$$

where

$$
\gamma:=\left\|\frac{1}{\sigma s-H^{T} A_{22} H} A_{12} H H^{T} A_{21}\left(s I_{m}-A_{11}\right)^{-1}\right\|_{H}
$$

and $\|\cdot\|_{H_{\infty}}$ means $H$-infinity norm of the transfer function.

Proof: Notice that

$$
\|u(\cdot)\|_{\mathcal{L}_{2}} \leq\left\|\frac{1}{\sigma} A_{12} H y(\cdot)\right\|_{\mathcal{L}_{2}}+\|v(\cdot)\|_{\mathcal{L}_{2}} .
$$

Since $y(\cdot)$ is generated by $v(\cdot)$ through the transfer function

$$
\begin{gathered}
{\left[\begin{array}{ll}
0_{1 \times m} & 1
\end{array}\right]\left(s I-\left[\begin{array}{cc}
A_{11} & 0 \\
H^{T} A_{21} & \frac{1}{\sigma} H^{T} A_{22} H
\end{array}\right]\right)^{-1}\left[\begin{array}{c}
I_{m} \\
0
\end{array}\right]=} \\
\frac{1}{s-H^{T} A_{22} H / \sigma} H^{T} A_{21}\left(s I_{m}-A_{11}\right)^{-1}
\end{gathered}
$$

we have that

$$
\left\|\frac{1}{\sigma} A_{12} H y(\cdot)\right\|_{\mathcal{L}_{2}} \leq \gamma\|v(\cdot)\|_{\mathcal{L}_{2}} .
$$

where $\gamma$ is defined in (19). From this we can obtain the thesis.

Corollary 1 Let $\hat{\mathcal{W}}_{o}$ be the output controllability Gramian of the reduced system (15) and $\overline{\mathcal{W}}_{o}$ be the output controllability Gramian of the reduced system (17). Then

$$
\overline{\mathcal{W}}_{o} \leq(1+\gamma)^{2} \hat{\mathcal{W}}_{o}
$$

Proof: For any final target output $y_{f}$ it is possible to find the optimal input $v_{\text {opt }}(\cdot)$ such that the system (17) generates from the zero initial state a state $\hat{x}$ and an output such that $y(t)=y_{f}$. Let instead $u_{\text {opt }}(\cdot)$ be the optimal input that drives the system (14) from the zero initial to a state evolution such that $y(t)=y_{f}$. From Lemma 2 we know that

$$
\left\|v_{\text {opt }}(\cdot)\right\|_{\mathcal{L}_{2}}=\overline{\mathcal{W}}_{o}^{-1 / 2}\left|y_{f}\right| \quad\left\|u_{\text {opt }}(\cdot)\right\|_{\mathcal{L}_{2}}=\hat{\mathcal{W}}_{o}^{-1 / 2}\left|y_{f}\right| .
$$

Let $u(\cdot)$ be the input obtained from $\hat{x}, v$ by (16). If we apply it to the system (12) we obtain the same state evolution and output evolution. Therefore, by the previous lemma we argue that

$$
\begin{aligned}
& \hat{\mathcal{W}}_{o}^{-1 / 2}\left|y_{f}\right|=\left\|u_{\text {opt }}(\cdot)\right\|_{\mathcal{L}_{2}} \leq\|u(\cdot)\|_{\mathcal{L}_{2}} \leq \\
&(1+\gamma)\left\|v_{\text {opt }}(\cdot)\right\|_{\mathcal{L}_{2}} \leq(1+\gamma) \overline{\mathcal{W}}_{o}^{-1 / 2}\left|y_{f}\right|
\end{aligned}
$$

From this the result claimed is straightforward.

\section{A. The output controllability Gramian of the reduced system}

Let $\bar{A}, \bar{B}$ the system matrices in (17) with $\bar{C}=\hat{C}$ the output matrix. Thanks to the definition (16) and to the resulting block triangular structure of $\bar{A}$, the Gramian of the reduced system (17) can be computed explicitly. Lets simplify slightly the notation by introducing

$$
\bar{A}=\left[\begin{array}{cc}
A_{11} & 0 \\
a^{T} & \alpha
\end{array}\right],
$$

where $a^{T}=H^{T} A_{21}, \quad \alpha=1 / \sigma H^{T} A_{22} H$. In the following we will limit our analysis to infinite time controllability Gramians namely to the case in which $t_{f}=\infty$. We will assume moreover that $\bar{A}$ is Hurwitz stable, otherwise the infinite time controllability Gramian $\mathcal{W}$ associated with $\bar{A}, \bar{B}$ would not be well-defined. Observe that in this case $\overline{\mathcal{W}}$ has to satisfy the Lyapunov equation $\bar{A} \overline{\mathcal{W}}+\mathcal{W} \bar{A}^{T}+\bar{B} \bar{B}^{T}=0$. If we partition $\overline{\mathcal{W}}$ according to the block structure of $\bar{A}$ and $\bar{B}$ as

$$
\overline{\mathcal{W}}=\left[\begin{array}{ll}
\overline{\mathcal{W}}_{11} & \overline{\mathcal{W}}_{12} \\
\overline{\mathcal{W}}_{12}^{T} & \overline{\mathcal{W}}_{22}
\end{array}\right]
$$

then given the definition of $\bar{C}$, we have that $\overline{\mathcal{W}}_{o}=\overline{\mathcal{W}}_{22}$. Moreover, the previous Lyapunov equation is equivalent to the following three equations

$$
\begin{aligned}
& A_{11} \overline{\mathcal{W}}_{11}+\overline{\mathcal{W}}_{11} A_{11}^{T}+I_{m}=0 \\
& A_{11} \overline{\mathcal{W}}_{12}+\overline{\mathcal{W}}_{11} a+\overline{\mathcal{W}}_{12} \alpha=0 \\
& a^{T} \overline{\mathcal{W}}_{12}+\alpha \overline{\mathcal{W}}_{22}+\overline{\mathcal{W}}_{12}^{T} a+\alpha \overline{\mathcal{W}}_{22}=0 .
\end{aligned}
$$

From the second equation we argue that ${ }^{1} \overline{\mathcal{W}}_{12}=-\left(A_{11}+\right.$ $\left.\alpha I_{m}\right)^{-1} \overline{\mathcal{W}}_{11} a$ and, using the third equation, we have that

$$
\begin{aligned}
\overline{\mathcal{W}}_{22}= & \frac{1}{2 \alpha}\left[a^{T}\left(A_{11}+\alpha I_{m}\right)^{-1} \overline{\mathcal{W}}_{11} a\right. \\
& \left.\quad+a^{T} \overline{\mathcal{W}}_{11}\left(A_{11}^{T}+\alpha I_{m}\right)^{-1} a\right] \\
= & \frac{1}{2 \alpha} a^{T}\left(A_{11}+\alpha I_{m}\right)^{-1}\left[2 \alpha \overline{\mathcal{W}}_{11}-I_{m}\right]\left(A_{11}^{T}+\alpha I_{m}\right)^{-1} a \\
= & a^{T} \Phi a,
\end{aligned}
$$

where

$$
\Phi:=\frac{1}{2 \alpha}\left(A_{11}+\alpha I_{m}\right)^{-1}\left[2 \alpha \overline{\mathcal{W}}_{11}-I_{m}\right]\left(A_{11}^{T}+\alpha I_{m}\right)^{-1} .
$$

This implies that $\overline{\mathcal{W}}_{o} \leq\|\Phi\| a^{T} a$. Notice that, if $A_{11}$ is symmetric, then $\overline{\mathcal{W}}_{11}=-\left(2 A_{11}\right)^{-1}$ and so

$$
\begin{aligned}
\Phi & =\frac{1}{2 \alpha}\left(A_{11}+\alpha I_{m}\right)^{-1}\left[-\alpha A_{11}^{-1}-I_{m}\right]\left(A_{11}+\alpha I_{m}\right)^{-1} \\
& =-\frac{1}{2 \alpha}\left(A_{11}+\alpha I_{m}\right)^{-1} A_{11}^{-1} .
\end{aligned}
$$

${ }^{1}$ Notice that, since $\bar{A}$ is Hurwitz stable, then $\alpha<0$. Hence Hurwitz stability of $A_{11}$ implies that $A_{11}+\alpha I_{m}$ is invertible. 
We can observe that:

i) $a^{T} a$ is proportional to the connectivity degree between the controlled nodes and the output, i.e.

$$
a^{T} a \propto d_{z \rightarrow \delta},
$$

where $d_{z \rightarrow \delta}$ is the out-degree from controlled nodes $z$ to aggregated nodes $\delta$;

ii) $\Phi$ can be seen as the network viscosity of the aggregated dynamics and the controlled nodes. Its norm is given as follows

$$
\|\Phi\|=\frac{1}{-2 \alpha^{2} \lambda_{\max }\left(A_{11}\right)-2 \alpha \lambda_{\max }\left(A_{11}\right)^{2}},
$$

where $\lambda_{\max }\left(A_{11}\right)$ is the largest eigenvalue of $A_{11}$.

From i), we can infer that controllability depends on the number of connections between controlled nodes and the aggregated nodes: the more connections, the less energy required to control the output of the aggregated nodes. From ii), we observe that the smaller the time-constant of the aggregated nodes $\alpha$ and $\lambda_{\max }\left(A_{11}\right)$, the bigger $\Phi$ and thus the less the energy required to control the output of the aggregated nodes.

It is worth pointing out that (21)-(22) can only be obtained for the reduced system (17): for the original system (10), the explicit computation of the Gramian is in general impossible. One of the advantages of considering the reduced system (17) is thus to enable an analytic study of the Gramian which in turns allows to assess the impact that the parameters of the network (such as connectivity and degrees) have on the energy necessary to control the system output.

\section{B. Application to Large-Scale Networks}

We are now interested in comparing $\mathcal{W}_{o}$ with $\hat{\mathcal{W}}_{o}$ in the case of a large-scale network. We consider networks in which we fix the number of uncontrolled nodes $\eta=500$ and we vary the number $m$ of controlled boundary nodes (namely nodes with a low betweenness centrality). The network dynamics is described by equation (10) where $A$ is a Hurwitz Metzler matrix (see also [27]). The graph has been generated with a Watts-Strogatz model with mean degree 4 (inspired by Manhattan transportation grids).

We set $H^{T}=\frac{1}{\eta} \mathbb{1}^{T}$, namely we assume that $y$ is the average of the uncontrolled nodes. This choice is motivated by transportation networks where typically we are allowed to control the boundaries of the graph with the goal to steer some measure of the interior nodes (in this case the average) to a desired value [34].

In order to compare $\hat{\mathcal{W}}_{o}$ for the reduced system (15) and $\mathcal{W}_{o}$ for the original system (10), we compute the optimal input for the original system (10)

$$
u_{\text {out }}^{o}(t)=B^{T} e^{A^{T}\left(t_{f}-t\right)} C^{T} \mathcal{W}_{o}^{-1} y_{f},
$$

and for the reduced system (15)

$$
\hat{u}_{\text {out }}^{o}(t)=\hat{B}^{T} e^{\hat{A}^{T}\left(t_{f}-t\right)} \hat{C}^{T} \hat{\mathcal{W}}_{o}^{-1} y_{f},
$$

where we assumed that the initial state is zero. We set $y_{f}=1$ and $t_{f}=10 \mathrm{sec}$.

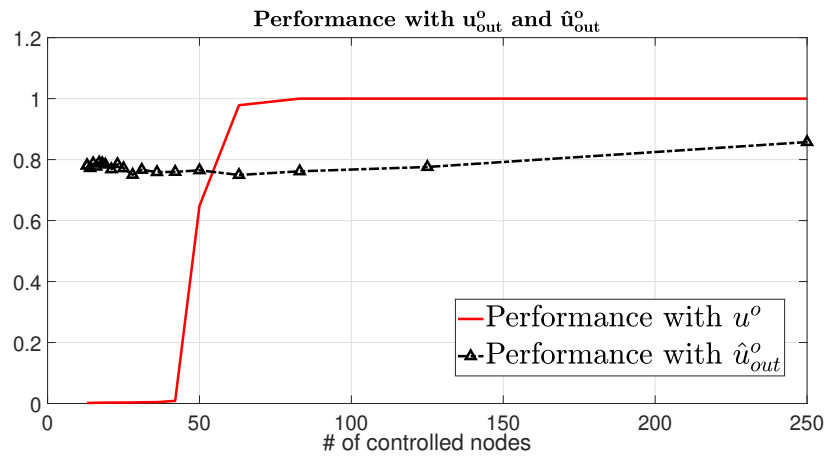

Fig. 2: Performance of the system for $n=500$ and decreasing values of $m$, with two different control inputs $u^{o}$ and $\hat{u}^{o}$, respectively calculated on the original system and on the reduced system for different size of the network.

Then we apply the two inputs $u_{\text {out }}^{o}(t)$ and $\hat{u}_{\text {out }}^{o}(t)$ to the same system (10) and evaluate the output at time $t_{f}$. Let $y\left(t_{f}, u_{\text {out }}^{o}\right)$ and $y\left(t_{f}, \hat{u}_{\text {out }}^{o}\right)$ be these two outputs. To compare the two inputs we then consider the following ratios

$$
\frac{y\left(t_{f}, u_{\text {out }}^{o}\right)}{y_{f}}, \quad \frac{y\left(t_{f}, \hat{u}_{\text {out }}^{o}\right)}{y_{f}} .
$$

Figure 2 shows the comparison of the two ratios (25) for different values of $m=\left\{\frac{\eta}{2}, \ldots, \frac{\eta}{20}\right\}$. As long as the $m \geq \frac{1}{5} \eta$, the control input (23) (red plot) outperforms (24) (black plot) by around $20 \%$. However, we find out that around the ratio $m=\frac{1}{6} \eta$ a transition appears in the controllability properties of a network. In particular the performance when considering input (23) degrades abruptly for $m \leq \frac{1}{6} \eta$. This phenomenon does not appear when we design the input as (24) exploiting the reduced system. This result is mainly due to the fact that the computation of $\mathcal{W}_{o}$ in (23) becomes highly illconditioned for small $m$ while $\hat{\mathcal{W}}_{o}$ in (24), though being an approximation, is a scalar.

Furthermore, to confirm the conclusions drawn in Section III-A, we considered the same network with a fixed number of controllers $m=100$. In Figure 3, we plot the ratios (25) and the control energy with the two inputs (23) and (24) respectively, for increasing connectivity degrees $d_{z \rightarrow \delta}$. In Figure 4, we do the same by letting $\|\Phi\|$ in (22) vary. Figure 3 and Figure 4 show that the network structure (through $d_{z \rightarrow \delta}$ and $\Phi$ ) has a real impact on the control energy. This is well captured by the reduced system (15) being a reliable energy approximation of the original system.

\section{A REDUCED MODEL NETWORK: THE CASE OF MULTIPLE OUTPUTS}

In many practical cases, we are interested in considering multiple outputs describing the evolution of a network. This aspect is strictly related to the possibility of partitioning a network in multiple zones and to associate a measure to each. In the context of transportation network for instance, these partitions might represent areas/districts we are interested in monitoring and controlling. In this section, the network is partitioned according to the outputs and the controlled nodes, sitting on the boundary, associated to each output. In Figure 1 


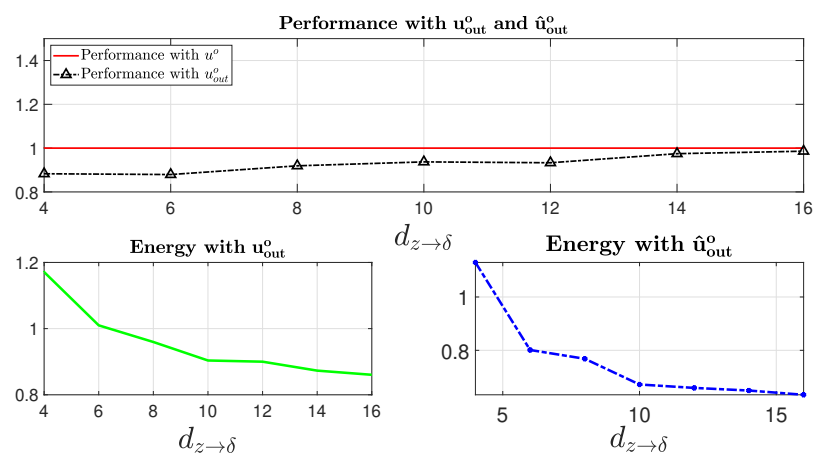

Fig. 3: Performance and energy computed with respect to the connectivity degree in (21). The control energy decreases for an increasing $d_{z \rightarrow \delta}$.

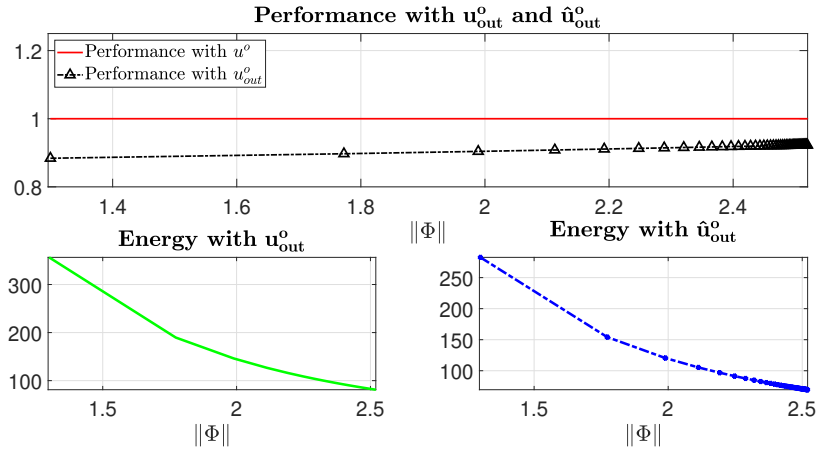

Fig. 4: Performance and energy with respect to inertia of the network $\|\Phi\|$ in (22). Again, the control energy decreases for an increasing $\|\Phi\|$.

an example of this setting is shown: the network is partitioned according to 4 measured outputs, each partition with its own set of controlled boundary nodes, each partition potentially interacting with the others.

We consider again a system in the form (9) where $A \in$ $\mathbb{R}^{n \times n}$ is an Hurwitz Meltzer matrix, $B$ is an input $m \times$ $n$ matrix with entries in $\{1,0\}$ which defines which nodes are controlled and $C \in \mathbb{R}^{r \times n}$ describes the $r$ outputs to be controlled. We assume that each $y_{i}$ depends on a set of uncontrolled nodes and that these sets are disjoint for each output. More precisely, if we define $\mathcal{K} \subseteq \mathcal{V}=\{1,2, \ldots, n\}$ as the set of controlled nodes, then we can partition $\mathcal{V} \backslash \mathcal{K}$ in disjoint sets $\mathcal{S}_{1}, \mathcal{S}_{2}, \ldots, \mathcal{S}_{r}$ such that

$$
y_{i}=\sum_{j \in \mathcal{S}_{i}} C_{i j} x_{j} .
$$

If we partition the set of the controlled nodes $\mathcal{K}$ in $r$ disjoint subsets ${ }^{2} \mathcal{K}_{1}, \mathcal{K}_{2}, \ldots, \mathcal{K}_{r}$ and we define $\mathcal{V}_{i}:=\mathcal{S}_{i} \cup \mathcal{K}_{i}$, we can write each partition dynamics as

$\left[\begin{array}{c}\dot{z}_{i} \\ \dot{\delta}_{i}\end{array}\right]=\left[\begin{array}{cc}A_{11}^{i} & A_{12}^{i} \\ A_{21}^{i} & A_{22}^{i}\end{array}\right]\left[\begin{array}{c}z_{i} \\ \delta_{i}\end{array}\right]+\left[\begin{array}{c}I_{m_{i}} \\ 0\end{array}\right] u_{i}+\sum_{j \neq i}\left[\begin{array}{cc}R_{i j} & S_{i j} \\ T_{i j} & U_{i j}\end{array}\right]\left[\begin{array}{c}z_{j} \\ \delta_{j}\end{array}\right]$

$$
y_{i}=\left[\begin{array}{ll}
0 & \mathbf{h}_{\mathbf{i}}^{T}
\end{array}\right]\left[\begin{array}{c}
z \\
\delta_{i}
\end{array}\right] \text {, }
$$

\footnotetext{
${ }^{2}$ There is some arbitrarity in this choice.
}

where the vectors $z_{i} \in \mathbb{R}^{m_{i}}$ and $u_{i} \in \mathbb{R}^{m_{i}}$ represent the state and the input of the nodes in $\mathcal{K}_{i}$ and the vector $\delta_{i} \in \mathbb{R}^{\eta_{i}}$ contains the state of the nodes in $\mathcal{S}_{i}$. As mentioned previously, each partition's output $y_{i}$ depends only on $\delta_{i}$ through the vector $\mathbf{h}_{i}^{T}$.

The last term in the dynamics (26) describes the coupling between the different partitions of the network. In [16], the authors consider the problem of selecting control nodes in such a way that only $R_{i j} \neq 0$, namely partitions were copled through the control nodes. If this is the case, decoupling the partions by opportunely acting on the control nodes is an easy task and the resulting Gramian has a block diagonal structure which eases its computation. In this paper, control nodes are fixed and setting on the boundary of the network: then the outputs to be controlled (or rather the nodes $\delta_{i} \in \mathcal{S}_{i}$ contributing to the output $y_{i}$ ) are coupled with the other outputs (or rather with the nodes $\delta_{j} \in \mathcal{S}_{j}$ contributing to the output $y_{j}$ ). In contrast with [16], this leads to coupling matrices $U_{i j} \neq 0$, namely a coupling between the different outputs (i.e. partitions) in the network which cannot be compensated directly by control.

The off-diagonal terms $S_{i j}, T_{i j}$ are not considered in the following. As far as $S_{i j}$ is concerned, it would be easy to compensate this coupling within the same framework of [16]. As far as $T_{i j}$ is concerned, to compensate this coupling the same procedure illustrated in the following can be implemented. Thus, for the sake of simplicity (in notation and design), we focus only on the inter-partitions coupling, namely $U_{i j} \neq 0$.

The entire network, namely the composition of the $r$ partitions (26) can be rewritten as

$$
\begin{aligned}
{\left[\begin{array}{c}
\dot{z} \\
\dot{\delta}
\end{array}\right] } & =\left[\begin{array}{ll}
A_{11} & A_{12} \\
A_{21} & A_{22}
\end{array}\right]\left[\begin{array}{l}
z \\
\delta
\end{array}\right]+\left[\begin{array}{c}
I_{m} \\
0
\end{array}\right] u, \\
y & =\left[\begin{array}{ll}
0_{r \times m} & H^{T}
\end{array}\right]\left[\begin{array}{l}
z \\
\delta
\end{array}\right]
\end{aligned}
$$

where $z:=\left(z_{1}, z_{2}, \ldots, z_{r}\right), \delta:=\left(\delta_{1}, \delta_{2}, \ldots, \delta_{r}\right)$,

$$
\begin{aligned}
& A_{11}=\operatorname{blkdiag}\left\{A_{11}^{i}\right\}, A_{12}=\operatorname{blkdiag}\left\{A_{12}^{i}\right\} \\
& A_{21}=\operatorname{blkdiag}\left\{A_{21}^{i}\right\}, A_{22}=\operatorname{blkdiag}\left\{A_{22}^{i}\right\}+U
\end{aligned},
$$

where

$$
U=\left[\begin{array}{cccc}
0 & U_{12} & \ldots & U_{1 r} \\
U_{21} & 0 & & U_{2 r} \\
\vdots & & \ddots & \vdots \\
U_{r 1} & \ldots & U_{r, r-1} & 0
\end{array}\right]
$$

represents the interaction between the different partitions of the system and where $H^{T} \in \mathbb{R}^{r \times(n-m)}$ is the matrix which describes the $r$ outputs defined as $H^{T}=\operatorname{blkdiag}\left\{\mathbf{h}_{i}^{T}\right\}$.

Following the same steps presented in Section III for the single output case, we obtain a reduced system which 
partition-wise reads as

$$
\begin{aligned}
{\left[\begin{array}{c}
\dot{z}_{i} \\
\dot{y}_{i}
\end{array}\right]=\left[\begin{array}{cc}
A_{11}^{i} & \frac{1}{\sigma_{i}} A_{12}^{i} \mathbf{h}_{i} \\
\mathbf{h}_{i}^{T} A_{21}^{i} & \frac{1}{\sigma_{i}} \mathbf{h}_{i}^{T} A_{22}^{i} \mathbf{h}_{i}
\end{array}\right]\left[\begin{array}{c}
z_{i} \\
y_{i}
\end{array}\right]+\left[\begin{array}{c}
I_{m_{i}} \\
0
\end{array}\right] u_{i} } \\
+ \\
+\left[\begin{array}{c}
A_{12}^{i}\left(I_{\eta_{i}}-\frac{1}{\sigma_{i}} \mathbf{h}_{i} \mathbf{h}_{i}^{T}\right) \\
\mathbf{h}_{i}^{T} A_{22}^{i}\left(I_{\eta_{i}}-\frac{1}{\sigma_{i}} \mathbf{h}_{i} \mathbf{h}_{i}^{T}\right)
\end{array}\right] \delta_{i} \\
+\sum_{j \neq i}\left[\begin{array}{cc}
0 & 0 \\
0 & \frac{1}{\sigma_{j}} \mathbf{h}_{i}^{T} U_{i j} \mathbf{h}_{j}
\end{array}\right]\left[\begin{array}{c}
z_{j} \\
y_{j}
\end{array}\right] \\
+\sum_{j \neq i}\left[\begin{array}{c}
0 \\
\mathbf{h}_{i}^{T} U_{i j}\left(I_{\eta_{j}}-\frac{1}{\sigma_{j}} \mathbf{h}_{j} \mathbf{h}_{j}^{T}\right)
\end{array}\right] \delta_{j} .
\end{aligned}
$$

Again we neglect the effect of $\delta_{i}$ and $\delta_{j}$ on the evolution of the outputs $y_{i}$, thus obtaining

$$
\begin{aligned}
{\left[\begin{array}{c}
\dot{z}_{i} \\
\dot{y}_{i}
\end{array}\right]=} & {\left[\begin{array}{cc}
A_{11}^{i} & \frac{1}{\sigma_{i}} A_{12}^{i} \mathbf{h}_{i} \\
\mathbf{h}_{i}^{T} A_{21}^{i} & \frac{1}{\sigma_{i}} \mathbf{h}_{i}^{T} A_{22}^{i} \mathbf{h}_{i}
\end{array}\right]\left[\begin{array}{c}
z_{i} \\
y_{i}
\end{array}\right]+\left[\begin{array}{c}
I_{m_{i}} \\
0
\end{array}\right] u_{i} } \\
& +\sum_{j \neq i}\left[\begin{array}{cc}
0 & 0 \\
0 & \frac{1}{\sigma_{j}} \mathbf{h}_{i}^{T} U_{i j} \mathbf{h}_{j}
\end{array}\right]\left[\begin{array}{c}
z_{j} \\
y_{j}
\end{array}\right]
\end{aligned}
$$

We define a local pre-compensation control as

$$
u_{i}=-\frac{1}{\sigma_{i}} A_{12}^{i} \mathbf{h}_{i} y_{i}+v_{i},
$$

which yields to local triangular reduced systems

$$
\begin{aligned}
{\left[\begin{array}{c}
\dot{z}_{i} \\
\dot{y}_{i}
\end{array}\right]=} & {\left[\begin{array}{cc}
A_{11}^{i} & 0 \\
\mathbf{h}_{i}^{T} A_{21}^{i} & \frac{1}{\sigma_{i}} \mathbf{h}_{i}^{T} A_{22}^{i} \mathbf{h}_{i}
\end{array}\right]\left[\begin{array}{c}
z_{i} \\
y_{i}
\end{array}\right]+\left[\begin{array}{c}
I_{m_{i}} \\
0
\end{array}\right] v_{i} } \\
& +\sum_{j \neq i}\left[\begin{array}{cc}
0 & 0 \\
0 & \frac{1}{\sigma_{j}} \mathbf{h}_{i}^{T} U_{i j} \mathbf{h}_{j}
\end{array}\right]\left[\begin{array}{c}
z_{j} \\
y_{j}
\end{array}\right]
\end{aligned}
$$

However, system (32) still presents a coupling between different outputs $y_{i}$ and $y_{j}$ of partitions $\mathcal{S}_{i}, \mathcal{S}_{j}$ through the interaction term $\frac{1}{\sigma_{j}} \mathbf{h}_{i}^{T} U_{i j} \mathbf{h}_{j}$. In order to decouple the partitions we define ${ }^{3}$

$$
v_{i}=-\left(\mathbf{h}_{i}^{T} A_{21}^{i}\right)^{+}\left\{\frac{\mathbf{h}_{i}^{T} A_{22}^{i} \mathbf{h}_{i}}{\sigma_{i}} d_{i}+f_{i}\right\}+\bar{v}_{i}
$$

where $d_{i}$ is the measured signal

$$
d_{i}:=\sum_{j \neq i} \frac{1}{\sigma_{j}} \mathbf{h}_{i}^{T} U_{i j} \mathbf{h}_{j} y_{j}
$$

and where $f_{i}$ is the output of the first order system

$$
\begin{aligned}
\dot{w}_{i} & =-\frac{1}{\tau} w_{i}+d_{i} \\
f_{i} & =-\frac{1}{\tau^{2}} w_{i}+\frac{1}{\tau} d_{i},
\end{aligned}
$$

where $\tau>0$.

Proposition 1 Consider the systems (30) and assume that the control input $u_{i}, i=1,2, \ldots, r$ is designed according to (31), (33), (34). Then, there exists a $\tau^{\star}$ such that, for any $\tau \leq \tau^{\star}$, the cascade (32), (33) and (34) is asymptotically stable for $\bar{v}_{i}=0$.

Proof: Observe first that, since the input and the output of (34) are related through the transfer function $\frac{s}{1+s \tau}$, then for

\footnotetext{
${ }^{3}$ Here, given a matrix $A \in \mathbb{R}^{m \times n}, A^{+}$denotes its Moore-Penrose inverse, namely $A A^{+}=I_{m}$.
}

small enough $\tau$ we have that $f_{i} \simeq \dot{d}_{i}$. Simple computations show that

$$
\begin{aligned}
\ddot{y}_{i}= & \left(\mathbf{h}_{i}^{T} A_{21}^{i} A_{11}^{i}+\frac{\mathbf{h}_{i}^{T} A_{22}^{i} \mathbf{h}_{i}}{\sigma_{i}} \mathbf{h}_{i}^{T} A_{21}^{i}\right) z_{i} \\
& +\left(\frac{\mathbf{h}_{i}^{T} A_{22}^{i} \mathbf{h}_{i}}{\sigma_{i}}\right)^{2} y_{i}+\frac{\mathbf{h}_{i}^{T} A_{22}^{i} \mathbf{h}_{i}}{\sigma_{i}} d_{i}+\dot{d}_{i}+\mathbf{h}_{i}^{T} A_{21}^{i} v_{i} \\
\simeq & \left(\mathbf{h}_{i}^{T} A_{21}^{i} A_{11}^{i}+\frac{\mathbf{h}_{i}^{T} A_{22}^{i} \mathbf{h}_{i}}{\sigma_{i}} \mathbf{h}_{i}^{T} A_{21}^{i}\right) z_{i} \\
& +\left(\frac{\mathbf{h}_{i}^{T} A_{22}^{i} \mathbf{h}_{i}}{\sigma_{i}}\right)^{2} y_{i}+\mathbf{h}_{i}^{T} A_{21}^{i} \bar{v}_{i}
\end{aligned}
$$

We see in this way that we have compensated the coupling effect between $y_{i}$ and $y_{j}$ from neighbor partitions $\mathcal{S}_{j}$. The existence of a finite $\tau^{\star}$ is guaranteed by the fact that $A$ is Meltzer Hurwitz and as a consequence, by definition of the projection matrix $P$ in (11), all matrixes

$$
\left[\begin{array}{cc}
A_{11}^{i} & 0 \\
\mathbf{h}_{i}^{T} A_{21}^{i} & \frac{1}{\sigma_{i}} \mathbf{h}_{i}^{T} A_{22}^{i} \mathbf{h}_{i}
\end{array}\right]
$$

with $i=1,2, \ldots, r$ are Hurwitz as well (see [35] for more details on the design of extended high gain observers). Then, following Proposition 4.7.2 in [36], the claim of the proposition follows by selecting opportunely $\tau \leq \tau^{\star}$ sufficiently small.

As a consequence of Proposition 1, when $\bar{v}_{i} \neq 0$, we can rewrite (32) as

$$
\left[\begin{array}{c}
\dot{z}_{i} \\
\dot{y}_{i}
\end{array}\right]=\left[\begin{array}{cc}
A_{11}^{i} & 0 \\
\mathbf{h}_{i}^{T} A_{21}^{i} & \frac{\mathbf{h}_{i}^{T} A_{22}^{i} \mathbf{h}_{i}}{\sigma_{i}}
\end{array}\right]\left[\begin{array}{c}
z_{i} \\
y_{i}
\end{array}\right]+\left[\begin{array}{c}
I_{m_{i}} \\
0
\end{array}\right] \bar{v}_{i} .
$$

Hence, to steer the output of the partition $\mathcal{S}_{i}$ to its desired value, the control energy can be written as a function of three contributions

$$
\begin{gathered}
\left\|u_{i}(\cdot)\right\|_{\mathcal{L}_{2}} \leq \| \\
\quad \frac{1}{\sigma_{i}} A_{12}^{i} \mathbf{h}_{i} y_{i}(\cdot)\left\|_{\mathcal{L}_{2}}+\right\| \bar{v}_{i}(\cdot) \|_{\mathcal{L}_{2}} \\
+\left\|M_{i} d_{i}+N_{i} f_{i}\right\|_{\mathcal{L}_{2}},
\end{gathered}
$$

where $M_{i}, N_{i}$ are defined as

$$
M_{i}=\left(\mathbf{h}_{i}^{T} A_{21}^{i}\right)^{+} \frac{\mathbf{h}_{i}^{T} A_{22}^{i} \mathbf{h}_{i}}{\sigma_{i}}, \quad N_{i}=\left(\mathbf{h}_{i}^{T} A_{21}^{i}\right)^{+} .
$$

From the same arguments used in Lemma 3, we have

$$
\left\|\frac{1}{\sigma_{i}} A_{12}^{i} \mathbf{h}_{i} y_{i}(\cdot)\right\|_{\mathcal{L}_{2}} \leq \gamma_{i}\left\|\bar{v}_{i}(\cdot)\right\|_{\mathcal{L}_{2}},
$$

where

$$
\gamma_{i}:=\left\|\frac{1}{\sigma_{i} s-\mathbf{h}_{i}^{T} A_{22} \mathbf{h}_{i}} A_{12}^{i} \mathbf{h}_{i} \mathbf{h}_{i}^{T} A_{21}^{i}\left(s I_{m_{i}}-A_{11}^{i}\right)^{-1}\right\|_{H_{\infty}}
$$

and where $\|\cdot\|_{H_{\infty}}$ means $H_{\infty}$ norm of the transfer function. Define $\delta_{j}, \gamma_{i j}$ respectively as

$$
\begin{aligned}
\delta_{j} & :=\left\|\frac{1}{s-\frac{1}{\sigma_{j}} \mathbf{h}_{j}^{T} A_{22}^{j} \mathbf{h}_{j}} \mathbf{h}_{j}^{T} A_{21}^{j}\left(s I_{m_{i}}-A_{11}^{j}\right)^{-1}\right\|_{H_{\infty}} \\
\gamma_{i j} & :=\left\|\frac{1}{\sigma_{j}} \mathbf{h}_{i}^{T} U_{i j} \mathbf{h}_{j}\right\|
\end{aligned}
$$


and observe that the $H_{\infty}$ norm of the transfer function $\frac{s}{1+s \tau}$ is $1 / \tau$. Then

$$
\left\|M_{i} d_{i}+N_{2} f_{i}\right\|_{\mathcal{L}_{2}} \leq\left(\left\|M_{i}\right\|+\frac{1}{\tau}\left\|N_{i}\right\|\right) \sum_{j=1}^{r} \gamma_{i j} \delta_{j}\left\|\bar{v}_{j}(\cdot)\right\|_{\mathcal{L}_{2}} .
$$

We now set $\delta_{i j}:=\left(\left\|M_{i}\right\|+\frac{1}{\tau}\left\|N_{i}\right\|\right) \gamma_{i j} \delta_{j}$ and let $\Delta$ be the $r \times r$ matrix with entries $\delta_{i j}$. Let moreover $\Gamma$ be the $r \times r$ diagonal matrix with entries $1+\gamma_{i}$. Observe that there exists $\bar{v}_{i}(\cdot)$ driving the system (35) from the zero state to the final output $y_{i f}$ and such that

$$
\left\|\bar{v}_{i}(\cdot)\right\|_{\mathcal{L}_{2}}=\overline{\mathcal{W}}_{o i}^{-1 / 2}\left|y_{i f}\right|,
$$

where $\overline{\mathcal{W}}_{o i}$ is the output controllability Gramian of the system (35).

Then, the following result is straightforward.

Lemma 4 Let $\bar{v}_{i}, i=1,2, \ldots, r$, be the inputs generating the state evolutions $\hat{x}_{i}, i=1,2, \ldots, r$, of the $r$ systems (30) and let $u_{i}$ in (31) be generated from these $\hat{x}_{i}, v_{i}$. Then

$$
\begin{aligned}
\|u(\cdot)\|_{\mathcal{L}_{2}}^{2} & =\sum_{i}\left\|u_{i}(\cdot)\right\|_{\mathcal{L}_{2}}^{2} \\
& \leq y_{f}^{T} \Lambda^{1 / 2}(\Gamma+\Delta)^{T}(\Gamma+\Delta) \Lambda^{1 / 2} y_{f},
\end{aligned}
$$

where

$$
\Lambda:=\operatorname{diag}\left\{\overline{\mathcal{W}}_{o 1}^{-1}, \overline{\mathcal{W}}_{o 2}^{-1}, \ldots, \overline{\mathcal{W}}_{o r}^{-1}\right\} .
$$

With (40) in mind, energy properties of the overall networks can be upper-bounded by means of the analysis performed in Section III-A for the each block of the matrix $\Lambda$ and by considering the energy required to decouple the partitions. The latter depends on the $\mathcal{L}_{2}$-gain matrix $\Gamma+\Delta$ which in turns depends on the coupling terms between partitions through (37), (38) and on the design of the observer (34) through (39).

Last, we introduce a corollary that puts in relationship the output controllability Gramian $\hat{\mathcal{W}}_{o}$ of (30) and the output controllability Gramians $\overline{\mathcal{W}}_{o i}$ of (35).

Corollary 2 Let $\hat{\mathcal{W}}_{o}$ be the output controllability Gramian of the reduced system (30) and $\overline{\mathcal{W}}_{o i}$ be the output controllability Gramians for the $r$ partitions (35). Then

$$
\left\|\hat{\mathcal{W}}_{o}^{-1}\right\| \leq\|\Gamma+\Delta\|^{2}\|\Lambda\|=\|\Gamma+\Delta\|^{2} \max _{i}\left\{\overline{\mathcal{W}}_{o i}^{-1}\right\} .
$$

\section{A. Application to Large-Scale Networks}

We are again interested in comparing $\mathcal{W}_{o}$ of (27) with $\hat{\mathcal{W}}_{o}$ of the network of $r$ partitions (30), when the $r$ outputs have to be controlled. In this first simulation, we replicate the same setup of Section III-B, namely we consider the behavior of the network with a fixed number of uncontrolled nodes $\eta=500$ and vary the number of controlled nodes $m$, while varying the number of outputs $r=3,5,10$. The graph connecting the uncontrolled nodes is generated with a WattsStrogatz model of mean degree 4 and partitions contain an equal number of nodes (almost equal for $r=3$ ).
The quality measure for the multi-output case is defined as

$$
\frac{\left\|y\left(t_{f}, u_{\text {out }}^{o}\right)\right\|}{\left\|y_{f}\right\|}, \quad \frac{\left\|y\left(t_{f}, \hat{u}_{\text {out }}^{o}\right)\right\|}{\left\|y_{f}\right\|} .
$$

where $y_{f} \in \mathbb{R}^{r}$ is the set of reference values that are selected randomly, $u_{\text {out }}^{o}$ is computed according to (23) for system (27), while $\hat{u}_{\text {out }}^{o}$ is computed according to (24) for the system

$$
\dot{\hat{x}}=\hat{A} \hat{x}+\hat{B} u \text {. }
$$

which is obtained by grouping the $r$ dynamics (30) with $\hat{x}_{i}=$ $\left[z_{i}, y_{i}\right]^{T}, \hat{x}=\operatorname{col}\left(\hat{x}_{1}, \hat{x}_{2}, \ldots, \hat{x}_{r}\right), u=\operatorname{col}\left(u_{1}, u_{2}, \ldots, u_{r}\right)$.

In Figure 5 we can observe that for large values of $m$ the behavior of the system driven by $u_{\text {out }}^{o}$ is close to the behavior of the system driven by $\hat{u}_{\text {out }}^{o}$. The difference in performance is indeed less than $10 \%$. Furthermore the more partitions $r$ we have, the closer we get to the nominal behavior. Compared to the case of one output, having multiple partitions implies that more information about the network is preserved and thus a better performance is maintained with the reduced model (30). This, together with the robustness with respect to a decreasing number of controlled nodes, provides an interesting insight in the possibility to control a large-scale network via the proposed reduced model.

These aspects are even more visible when we observe the behavior of the reduced system with respect to an increasing size of the network. In this case, we fix the number of outputs to $r=3$, the number of controlled nodes to $m_{i}=30$ (for each of the 3 partitions) and let the size of the network increase: results are displayed in Figure 6. As we can observe, for a small network of $n=150$ nodes (where thus the majority are controlled), the performance of the reduced model is ideal. As the size increases, up to $n \approx 1000$ nodes, the decrease in performances is scarcely influenced by the size of the network. This aspect confirms the reliability of the proposed reduced model.

In Figure 7, we illustrate the impact of the coupling between partitions, i.e. $\frac{1}{\sigma_{j}} \mathbf{h}_{i}^{T} U_{i j} \mathbf{h}_{j}$ in (32), for a fixed number of outputs $r=10$ and a fixed ratio of controlled nodes $m_{i}=\frac{\eta_{i}}{5}$ for each partition. For an increasing number of connections between the partitions, we compute the energy necessary to control the system with (7) and compare it with the energy estimated with (40). We start from weakly interacting partitions (only 2 connections randomly selected), to strongly interacting partitions (11 connections between partitions of $n_{i}=50$ nodes). We can clearly observe that, the more connections between partitions we introduce, the more energy to control the outputs to their target value is required. This is reflected both in the nominal energy (computed through the original system Gramian, red plot) and through the reduced model with the bound (40) (black plot), where we explicitly aim to decouple the partitions. Clearly, the decoupling energy depends on the density of connections between partitions and the more connections are present, the more the bound (40) becomes conservative. 


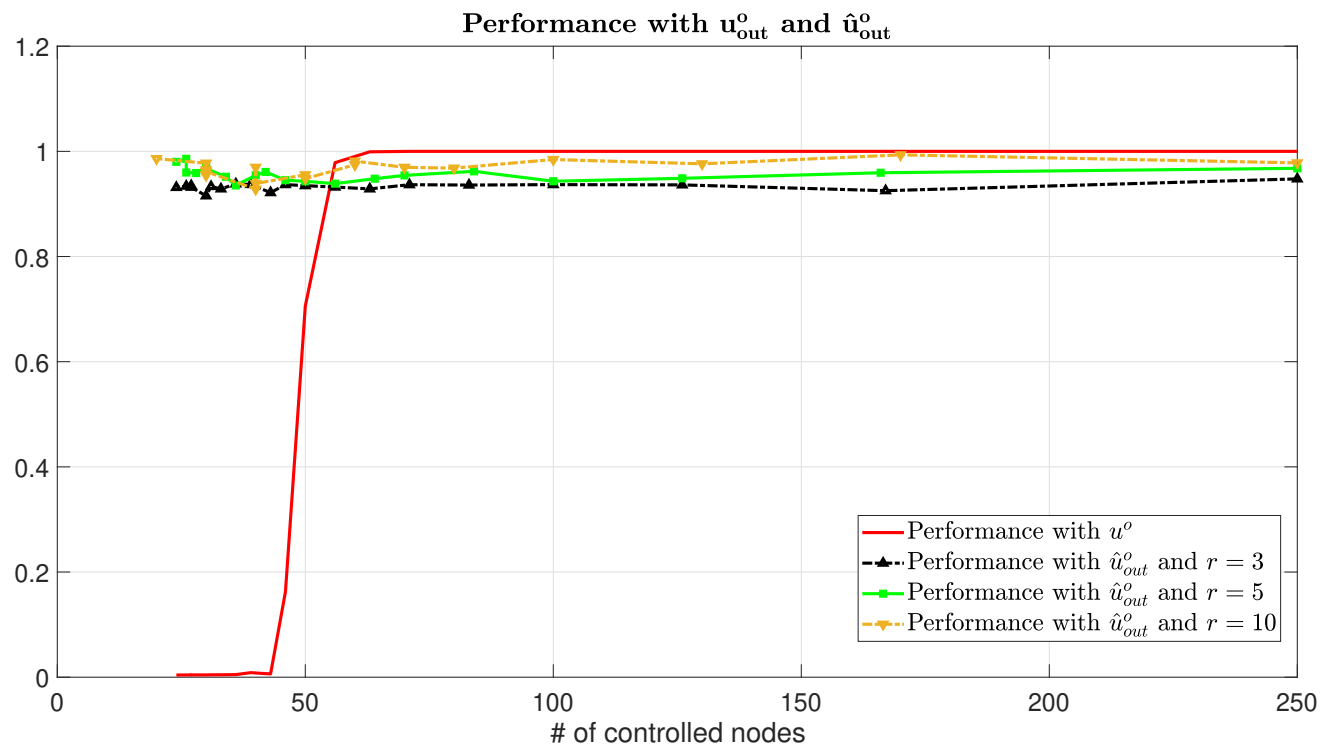

Fig. 5: Performance of the system for $\eta=500$, decreasing values of $m$ and $r=3,5,10$ partitions respectively, with two different control inputs $u^{o}$ and $\hat{u}^{\circ}$, calculated on the original system and on the reduced system respectively.

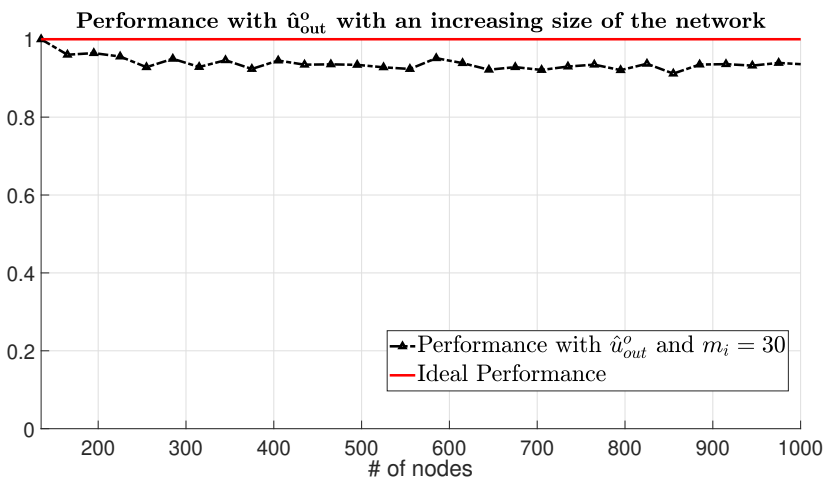

Fig. 6: Performance of the system for an increasing number of nodes $n$, a fixed number of outputs $r=3$ and a fixed number of controlled nodes per partition $m_{i}=30$.

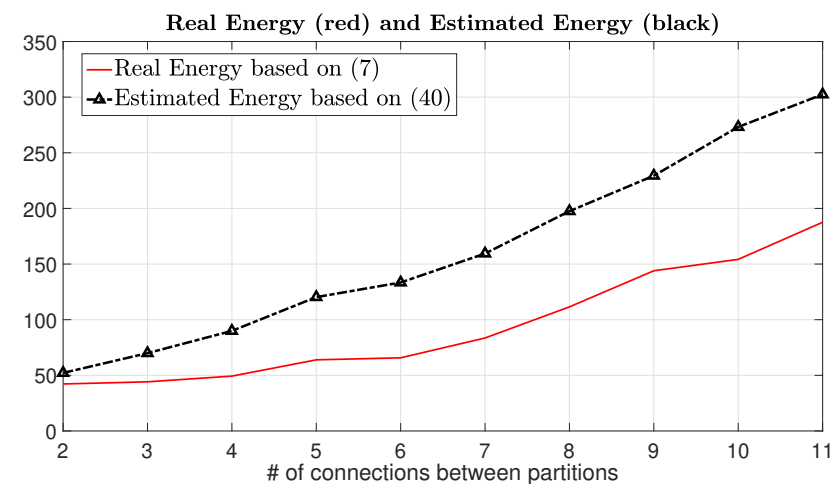

Fig. 7: Control energy as a function of the number of connections between partitions, for a fixed number of controlled nodes $m_{i}=$ $\eta_{i} / 5$ for each partitions. In red, the real energy calculated according to (7), in black the bound obtained in (40).

\section{CONCLUSIONS}

In this paper we considered the problem of controlling a large-scale network. Rather than controlling the full state, we considered the case in which we are interested in controlling an output, which depends on the nodes that are not directly controlled. To simplify the computation of the controllability Gramian (and eventually of the minimum energy control inputs), we considered an approximated model of the network, that is based on an aggregation of the uncontrolled nodes. This aggregation is defined as a projection of the output matrix. We showed that the reduced model is closed to the original network and in particular, the energy necessary to control the outputs is well approximated, even for small ratios of controlled nodes.

In the near future we plan to better understand the relationship between the partitions/outputs and the error introduced in the computation of the Gramian. In particular, simulation results show that for outputs that represent the state average of the nodes belonging to the each partition, the approximation is quite reliable. For a different choice of output, for instance a normal distribution around the average, the results are confirmed: yet it would be interesting to quantify the relationship between degree of asymmetry in the output and the error in the Gramian approximation.

We also plan to relax some assumption on the system dynamics (1), for instance the Hurwitz requirement. Another interesting development would be to consider more complex dynamics at the nodes (i.e. higher orders linear systems) and different coupling terms between the nodes.

\section{REFERENCES}

[1] G. Casadei, C. C. de Wit, and S. Zampieri, "Controllability of largescale networks: An output controllability approach," in IEEE Conf. on Decision and Control, 2018, pp. 5886-5891.

[2] R. Olfati-Saber, "Flocking for multi-agent dynamic systems: Algorithms and theory," IEEE Transactions on Automatic Control, vol. 51, no. 3, pp. 401-420, 2006.

[3] F. Dorfler and F. Bullo, "Synchronization and transient stability in power networks and nonuniform kuramoto oscillators," SIAM Journal on Control and Optimization, vol. 50, no. 3, pp. 1616-1642, 2012. 
[4] A. Mirtabatabaei and F. Bullo, "Opinion dynamics in heterogeneous networks: Convergence conjectures and theorems," SIAM Journal on Control and Optimization, vol. 50, no. 5, pp. 2763-2785, 2012.

[5] L. Moreau, "Stability of continuous-time distributed consensus algorithms," in IEEE Conf. on Decision and Control, 2004, pp. 3998-4003.

[6] P. Wieland, R.Sepulchre, and F. Allgöwer, "An internal model principle is necessary and sufficient for linear output synchronization," Automatica, vol. 47, no. 5, pp. 1068-1074, 2011.

[7] G. B. Stan and R. Sepulchre, "Analysis of interconnected oscillators by dissipativity theory," IEEE Transactions on Automatic Control, vol. 52, no. 2, pp. 256-270, 2007

[8] A. Isidori, L. Marconi, and G. Casadei, "Robust output synchronization of a network of heterogeneous nonlinear agents via nonlinear regulation theory," IEEE Transactions on Automatic Control, vol. 59, no. 10, pp. 2680-2692, 2014.

[9] M. Newman, A. Barabási, and D. Watts, The structure and dynamics of networks. Princeton University Press, 2006.

[10] M. E. J. Newman, "The structure and function of complex networks," SIAM Review, vol. 45, no. 2, pp. 167-256, 2003.

[11] R. Albert and A.-L. Barabási, "Statistical mechanics of complex networks," Reviews of Modern Physics, vol. 74, no. 1, pp. 47-97, 2002.

[12] R. E. Kalman, Y. C. Ho, and S. K. Narendra, "Controllability of linear dynamical systems," Contributions to Differential Equations, vol. 1, no. 2, pp. 189-213, 1963.

[13] C.-T. Lin, "Structural controllability," IEEE Transactions on Automatic Control, vol. 19, no. 3, pp. 201-208, 1974

[14] N. J. Cowan, E. J. Chastain, D. A. Vilhena, J. S. Freudenberg, and C. T. Bergstrom, "Nodal dynamics, not degree distributions, determine the structural controllability of complex networks," PloS one, vol. 7, no. 6, 2012.

[15] T. Zhou, "Minimal inputs/outputs for a networked system," IEEE Control Systems Letters, vol. 1, no. 2, pp. 298-303, 2017.

[16] F. Pasqualetti, S. Zampieri, and F. Bullo, "Controllability metrics, limitations and algorithms for complex networks," IEEE Transactions on Control of Network Systems, vol. 1, no. 1, pp. 40-52, 2014.

[17] G. Yan, G. Tsekenis, B. Barzel, J.-J. Slotine, Y.-Y. Liu, and A.-L. Barabási, "Spectrum of controlling and observing complex networks," Nature Physics, vol. 11, no. 9, p. 779, 2015.

[18] G. Lindmark and C. Altafini, "A driver node selection strategy for minimizing the control energy in complex networks," IFAC-PapersOnLine, vol. 50, no. 1, pp. 8309-8314, 2017.

[19] N. Bof, G. Baggio, and S. Zampieri, "On the role of network centrality in the controllability of complex networks," IEEE Transactions on Control of Network Systems, vol. 4, no. 3, pp. 643-653, 2017.

[20] G. Lindmark and C. Altafini, "Combining centrality measures for control energy reduction in network controllability problems," in European Control Conference, 2019, pp. 1518-1523.

[21] T. Kailath, Linear Systems. Prentice-Hall, 1980.

[22] V. Simoncini, "Computational methods for linear matrix equations," SIAM Review, vol. 58, no. 3, pp. 377-441, 2016.

[23] T. Ishizaki, K. Kashima, J. Imura, and K. Aihara, "Model reduction and clusterization of large-scale bidirectional networks," IEEE Transactions on Automatic Control, vol. 59, no. 1, pp. 48-63, 2014.

[24] N. Monshizadeh, H. L. Trentelman, and M. K. Camlibel, "Projectionbased model reduction of multi-agent systems using graph partitions," IEEE Transactions on Control of Network Systems, vol. 1, no. 2, pp. 145-154, 2014.

[25] M. S. Andersen, S. K. Pakazad, A. Hansson, and A. Rantzer, "Robust stability analysis of sparsely interconnected uncertain systems," IEEE Transactions on Automatic Control, vol. 59, no. 8, pp. 2151-2156, 2014.

[26] T. Zhou, "On the controllability and observability of networked dynamic systems," Automatica, vol. 52, pp. 63-75, 2015.

[27] A. Rantzer, "Scalable control of positive systems," European Journal of Control, vol. 24, pp. 72-80, 2015.

[28] D. Raczyński and W. Stanisławski, "Controllability and observability gramians parallel computation using gpu," Journal of Theoretical and Applied Computer Science, vol. 6, no. 1, pp. 47-66, 2012.

[29] J. Sun and A. E. Motter, "Controllability transition and nonlocality in network control," Physical review letters, vol. 110, no. 20, 2013.

[30] E. Kreindler and P. E. Sarachik, "On the concepts of controllability and observability of linear systems," IEEE Transactions on Automatic Control, vol. 9, no. 2, pp. 129-136, 1964.

[31] D. G. Luenberger, Optimization by Vector Space Methods. John Wiley, 1969.
[32] S. Boyd and C. Barratt, Linear Controller Design: Limits of Performance. Prentice Hall, 1991.

[33] M. Aoki, "Control of large-scale dynamic systems by aggregation," IEEE Transactions on Automatic Control, vol. 13, no. 3, pp. 246-253, 1968.

[34] M. U. B. Niazi, C. C. de Wit, and A. Kibangou, "Average observability of large-scale network systems," in European Control Conference, 2019, pp. 1-6.

[35] H. K. Khalil, "Cascade high-gain observer for high-dimensional systems," in IEEE Conf. on Decision and Control, 2016.

[36] A. Isidori, Nonlinear Control Systems, 3rd ed., ser. Communications and Control Engineering Series. Springer Verlag, 1995.

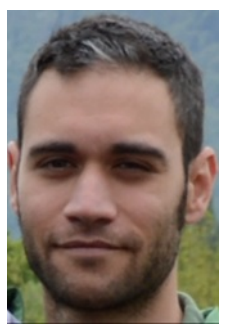

Giacomo Casadei is an associate professore in Ecole Centrale de Lyon at the Departement of Électronique - Électrotechnique - Automatique (E.E.A.) and in Laboratoire Ampère since 2018. He received the Master degree in Automation in 2012 and the Phd degree in Automation and Operative Research in 2016 at the University of Bologna (UNIBO). From 2016 to 2018, he has been postdoc in the NeCS team, a joint team of GIPSA-Lab (CNRS) and INRIA. His research interests include modeling and control of networks, with a particular focus on the problem of synchronization of nonlinear systems and the use of nonlinear control techniques in networks.

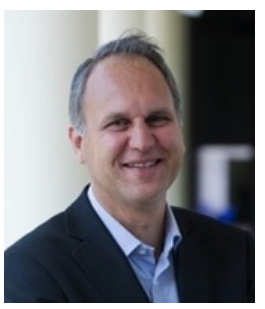

Carlos Canudas-de-Wit was born in Villahermosa, Mexico, in 1958. He received the B.S. degree in electronics and communications from the Monterrey Institute of Technology and Higher Education, Monterrey, Mexico, in 1980, and the M.S. and Ph.D. degrees in automatic control from the Department of Automatic Control, Grenoble Institute of Technology, Grenoble, France, in 1984 and 1987, respectively. He is currently a Directeur de recherche (Senior Researcher) with CNRS, Grenoble, where he is the Leader of the NeCS Team, a joint team of GIPSA-Lab (CNRS) and INRIA, on networked controlled systems. Dr. Canudas-de-Wit is an IEEE-CSS Fellow and an IFAC Fellow. $\mathrm{He}$ is an Associate Editor of the IEEE TRANSACTIONS ON AUTOMATIC CONTROL, the Automatica, the IEEE TRANSACTIONS ON CONTROL SYSTEMS TECHNOLOGY, the Asian Journal of Control, and the IEEE TRANSACTIONS ON CONTROL OF NETWORK SYSTEMS. He served as the President of the European Control Association from 2013 to 2015, and the IEEE Board of Governors of the Control System Society from 2011 to 2014. He holds the ERC Advanced Grant Scale-FreeBack from 2016 (19992002).

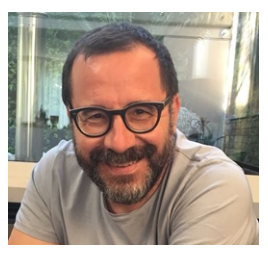

Sandro Zampieri Zampieri is Full Professor in Automatic Control at the Department of Information Engineering of the University of Padova since 2002. He received the Laurea degree in Electrical Engineering and the PhD degree in System Engineering from the University of Padova, Italy, in 1988 and 1993, respectively. His research interests include automatic control and dynamical systems theory, and in particular distributed control and estimation and networked control and control under communication constraints. He has delivered several invited seminars and he was member of the Technical Program Committee for several international conferences. He was general chair of the 1st IFAC Workshop on Estimation and Control of Networked Systems 2009, program chair of the 3rd IFAC Workshop on Estimation and Control of Networked Systems 2012 and publication chair of the IFAC World Congress 2011. He served as an Associate Editor of the Siam Journal on Control and Optimization on 20 02-20 04 and as the chair of the IFAC technical committee "Networked systems"on 2005-2008. Since 2012 he is serving as an Associate Editor of IEEE Transactions of Automatic Control. 